Type of the Paper: Article

\title{
Accelerating Gut Microbiome Research with Robust Sample Collection
}

\author{
Zoe J. Zreloff ${ }^{1}$, Danielle Lange ${ }^{1}$, Suzanne D. Vernon ${ }^{1}$, Martha R. Carlin ${ }^{1}$ and Raul J. Cano ${ }^{1 *}$ \\ 1 The BioCollective, 5650 Washington Street, Unit C9, Denver CO 80216; raul.cano@theBioCollective.com \\ * Correspondence: raul.cano@thebiocollective.com; Tel.: +1 805-748-9717
}

\begin{abstract}
:
Background. Inferior quality of biological material compromises data, slows discovery, and wastes research funds. The gut microbiome plays a critical role in human health and disease, yet little attention has been given to optimizing collection and processing methods of human stool.
\end{abstract}

Methods. We collected the entire bowel movement from 2 healthy volunteers: one to examine stool sample heterogeneity and one to test stool sample handling parameters. Sequencing and bioinformatic analyses were used to examine the microbiome composition.

Results. The microbiome profile varied depending on where the subsample was obtained from the stool. The exterior cortex of the stool was rich in specific phyla and deficient in others while the interior core of the stool revealed opposite microbiome profiles. Sample processing also resulted in varying microbiome profiles. Homogenization and stabilization at $4{ }^{\circ} \mathrm{C}$ gave superior microbial diversity profiles compared to the fresh or frozen subsamples of the same stool sample. Bacterial proliferation continued in the fresh subsample when processed at ambient temperature. Bacteroidetes proliferated and Firmicutes diminished during the 30-minute processing of fresh sample. The frozen sample had good overall diversity but Proteobacteria diminished likely because of the freeze/thaw.

Conclusions. The microbiome profile is specific to the section of the stool being sampled. Stool sample collection, homogenization, and stabilization at $4{ }^{\circ} \mathrm{C}$ for 24 hours provides a "neat", high-quality sample of sufficient quantity that can be banked into aliquots with nearly identical microbial diversity profiles. This collection pipeline is essential to accelerate our understanding of the gut microbiome in health and disease..

Keywords: Stool, Fecal, Microbiome, Microbiota,Heterogeneous, Homogeneous, Sample

\section{Introduction}

The more than 10 trillion microbial inhabitants of the gut are a valuable window into health and disease because of the myriad of interactions and influences these organisms have in our bodies. Accordingly, a representative and high-quality sample of the fecal microbiota is essential to advance gut microbiome research. Biological sample collection is the first and most important step in any research and development pipeline. If research testing begins with a sample that was not collected, processed or stored properly and is compromised in any way, the resulting data is unreliable and can lead further research astray or derail it completely.

Microbiome research has increased dramatically and is driven by advances in technology and decreases in sequencing costs. The majority of this research hinges on scientists' access to sufficient quantity of high-quality stool samples. While advanced sequencing technologies and sophisticated bioinformatics are used to decipher the human gut microbiota, little attention is paid to methods of collection, processing and storing of the stool sample that go into these sophisticated discovery pipelines. For example, two of 
the largest microbiome projects, American Gut Project and The Human Microbiome project used different fecal matter collection methods. Participants in the American Gut Project collected their fecal sample in the privacy of their home using a swab that was returned to the laboratory for sequencing via the United States Postal Service at ambient temperature [1]. The National Institutes of Health Human Microbiome Project had participants collect their stool sample in a plastic container which was then stored in a Styrofoam container with several frozen gel packs and returned within 24 hours of their bowel movement [2]. Other methods for collecting human stool for microbiome analysis involve using swabs [3-5], toilet paper wipes [6, 7], scoops [8], and containers to collect whole stools which can then be used in toto or subsampled with scoops or swabs [9, 10], Comparison of these sampling methods have shown disparity in microbiome composition [11, 12]. Standardization of sample collection was identified as one of the key knowledge gaps in microbiome research [11, 13-15].

Optimizing collection, processing, storage and preservation of human stool that is representative of the gut microbiome is essential for biomarker discovery. The objective of this study was to assess heterogeneity of the human stool and optimize collection and homogenization so that the microbiota remained viable and as representative as a recently evacuated stool sample for optimal use in many different omics platforms.

\section{Materials and Methods}

Sample Collection. The entire bowel movement was collected at the laboratory from two healthy volunteers using the BioCollector ${ }^{\mathrm{TM}}$ according to our IRB-approved protocol (IRB Tracking Number: 20160838). Both stools were Type 4 on Bristol Stool Form Scale [16]. To evaluate sample heterogeneity, the stool from one volunteer was dissected as shown in Figure 1. Samples weighing approximately one gram were taken at four different locations, one $\mathrm{cm}$ apart, along the length of the stool. Three sub-sections were each manually homogenized, aliquoted into two replicate aliquots, and stored at $-80^{\circ} \mathrm{C}$. The core of the fourth one-gram sample was separated from the cortex and each was independently homogenized, aliquoted, and stored. The remaining material from the stool was manually homogenized and aliquoted. DNA was extracted from the two replicate aliquots from each subsection of the dissected stool for sequencing (see below). To evaluate collection and processing, the stool from the second volunteer was emptied from the BioCollector ${ }^{\mathrm{TM}}$ into a plastic bag, closed and then thoroughly homogenized for 2 minutes by smashing and scraping using a plastic scraper. No homogenization buffer was used in this process. The homogenized, neat (nothing added) sample was divided into 3 equal subsamples for processing as follows: fresh (fresh); $4^{\circ} \mathrm{C}(4 \mathrm{C})$, and frozen on dry ice (frozen) (Figure 1). The $4 \mathrm{C}$ and frozen subsamples were handled first as follows; the $4 \mathrm{C}$ subsample was put into a mylar bag and on top of a frozen freezer brick in a Styrofoam container and stored for 24 hours. The frozen subsample was put into a mylar bag and placed in a Styrofoam container with dry ice for 24 hours. The fresh subsample was then processed over approximately 30 minutes into 80 cryovials containing 0.2 grams and frozen at $-80^{\circ} \mathrm{C}$ until sequencing. After 24 hours, the $4 \mathrm{C}$ subsample was aliquoted at room temperature into 20 cryovials each with 0.2 grams homogenized material and frozen at $-80^{\circ} \mathrm{C}$ until sequencing. The frozen subsample was thawed at $4^{\circ} \mathrm{C}$ for 24 hours and then aliquoted at room temperature into 20 cryovials each with 0.2 grams homogenized material and frozen at $-80^{\circ} \mathrm{C}$ until sequencing. 


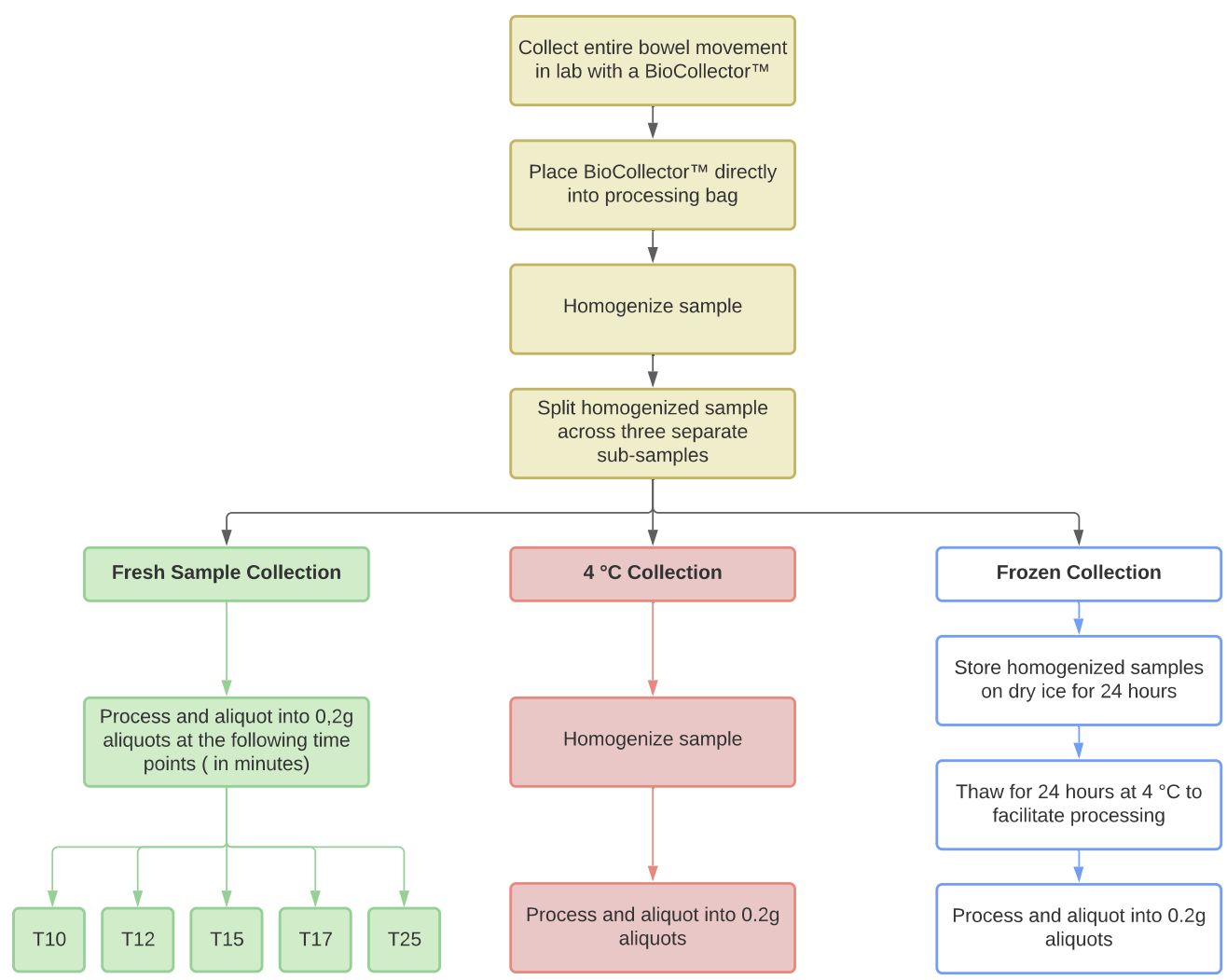

Figure 1. Sample Processing Workflow. This figure shows the steps taken in sample collection and processing for comparing sample preparation protocols

Library Prep and Sequencing. DNA from fecal samples was isolated using the QIAGEN DNeasy PowerSoil Pro Kit, according to the manufacturer's protocol. Isolated DNA was quantified by Qubit (ThermoFisher). A homogenized fecal reference material was included in all library preparations and sequencing runs. DNA libraries for whole genome sequencing were prepared using the Illumina Nextera XT library preparation kit, with a modified protocol. Library quantity was assessed with Qubit (ThermoFisher). Libraries were then sequenced on an Illumina HiSeq platform 2x150bp. The optimized $16 \mathrm{~S}$ sequencing covers the V3-V4 (341nt-805nt) region of the 16S rRNA gene with a two-step PCR strategy. The first step used the 16S-optimized primer set to amplify the V3-V4 regions of $16 \mathrm{~S}$ rDNA within the metagenomic DNA. The primer set contained optimized primers for comprehensive taxa coverage and frame shift primers for higher complexity. In addition to specific V3-V4 priming regions, these primers have sequences partially complementary to Illumina adapters. The first PCR amplifications were carried out in a $25 \mathrm{ul}$ volume. Each reaction mixture contained $2.5 \mu \mathrm{l} 1 \mathrm{X}$ primer mix, 5-50 ng metagenomic DNA, $0.5 \mu$ l AccuPrime Taq DNA Polymerase (ThermoFisher) and 2.5 10X AccuPrime PCR Buffer II (ThermoFisher). The PCR conditions included an initial denaturation step at $95^{\circ} \mathrm{C}$ for $2 \mathrm{~min}$, followed by 10 cycles of $95^{\circ} \mathrm{C}$ for $45 \mathrm{sec}, 57^{\circ} \mathrm{C}$ for $90 \mathrm{sec}, 72^{\circ} \mathrm{C}$ for $50 \mathrm{sec}$, and end with an extension step at $72^{\circ} \mathrm{C}$ for $10 \mathrm{~min}$. Next, the PCR products from the previous two reactions were mixed at equal amounts and used as templates in the second step to produce Illumina dual-index libraries for sequencing, with both adapters containing an 8-bp index allowing for multiplexing. Each reaction mixture contained $0.5 \mu \mathrm{l} \mathrm{Ac-}$ cuPrime Taq DNA Polymerase (ThermoFisher) and 2.5 10X AccuPrime PCR Buffer II (ThermoFisher), $3 \mu \mathrm{l} 10 \mu \mathrm{M}$ adapter primer D50x, $3 \mu \mathrm{l} 10 \mu \mathrm{M}$ adapter primer D70x, $4 \mu \mathrm{l}$ $10 \mu \mathrm{M}$ Illumina primer cocktail and $50 \mu \mathrm{l}$ PCR product from first PCR reaction mix. The 
PCR conditions included an initial denaturation step at $95^{\circ} \mathrm{C}$ for $2 \mathrm{~min}$, followed by $6 \mathrm{cy}-$ cles of $95^{\circ} \mathrm{C}$ for $45 \mathrm{sec}, 60^{\circ} \mathrm{C}$ for $30 \mathrm{sec}, 72^{\circ} \mathrm{C}$ for $50 \mathrm{sec}$, and end with an extension step at $72^{\circ} \mathrm{C}$ for $10 \mathrm{~min}$. The dual-indexed library amplification products were purified using Ampure beads (Beckman Coulter). Library quantification was performed using Qubit dsDNA HS assay (ThermoFisher) and qualified on a 2100 Bioanalyzer instrument (Agilent) to show a distribution with a peak in the expected range. A final qPCR quantification was performed before loading onto an MiSeq (Illumina) sequencer for PE250 (v2 chemistry). FastQC analysis of forward and reverse reads was conducted on all raw sequence data prior to use in analytic pipelines to determine the overall quality of the product and as a milestone prior to further analysis and to determine trim parameters for DADA2 [17]. The DADA2 script in QIIME2 [18] was executed for sequence quality control and feature table construction. This script removes and/or corrects reads with sequencing errors and removes chimeric.

Bioinformatic Analysis. All bioinformatics analysis was conducted using a QIIME2 version 2020.2 workflow similar to that described in QIIME2 for analyzing "Moving Pictures" data (https://docs.qiime2.org/2020.2/tutorials/moving-pictures/). Paired-end sequencing reads were imported into the workflow using Casava v1.8.2 d. Sequence quality control and features table construction was conducted using DADA2 [17]. Taxonomic analysis was conducted using the Silva [19] 132 99\% OTUs, full length, seven level taxonomy classifier (silva-132-99-nb-classifier.qza).

Quality Assurance. Two aliquots of a reference material derived from homogenized whole stools and fully characterized, both by metagenomics and metabolomic analysis were included, blindly, with the test fecal samples for process quality control. FastQC analysis of forward and reverse reads was conducted on all raw sequence data prior to use in analytic pipelines (https://www.bioinformatics.babraham.ac.uk/projects/fastqc/) to determine the overall quality of the product and as a milestone prior to further analysis and determine trim parameters for DADA2 [17]. The DADA2 script in QIIME2 [18] was executed for sequence quality control and feature table construction. This script removes and/or corrects reads with sequencing errors and removes chimeric.

Statistical analyses. All statistical analyses were performed using packages 'vegan' v2.5-6 and 'ggplot2' v3.3.2 in R 3.6.3 (https://www.r-project.org/). For microbiome analysis, rarefaction depth was set at 25,000 reads. Shannon diversity index. [20, 21], Chao1 Index [21] and Pielou's Evenness [22] were used to evaluate alpha (within sample) diversity. Beta (between sample) diversity was examined using multidimensional scaling analysis (MDA) [23] of Bray-Curtis [24] and Jaccard [25] distances. The Wilcoxon rank-sum test [26] was used to compare alpha diversity values between groups $(p>0.05)$. Statistical significance of beta-diversity distances between stool processing workflows was assessed using PERMANOVA [27] with 999 permutations. Alpha diversity group significance was calculated using nonparametric Kruskal-Wallis H test [28].

\section{Results}

Two aliquots of a reference material derived from homogenized whole stools were included in the batch submitted for sequencing to the test fecal samples for process quality control. Sequence count for both forward and reverse reads were $251 \mathrm{bp}$. The mean $\mathrm{Q}$ value for forward reads was 36 while that of the reverse reads was 34 . Based on these data the sequences were trimmed to $200 \mathrm{bp}$ prior to DADA2 analysis [32]. The average number of input-reads for DADA2 processing was 75,132 $\pm 6,052$, of which $37,175 \pm 5,556$ passes denoising and chimera analysis (Table S1). The "blind" reference sample passed QC based on taxonomic profile (Figure S1), Wilcoxson Rank Sum [41] Test (P-value = 0.3173 ) and Permutational Analysis of Variance (PERMANOVA) [42] (statistic $=0.989$, Pvalue $=0.532)$

To ascertain heterogeneity, the stool sample from one volunteer was dissected as shown in Figure 2. Sequencing depth was 57,720 $\pm 23,466$. 


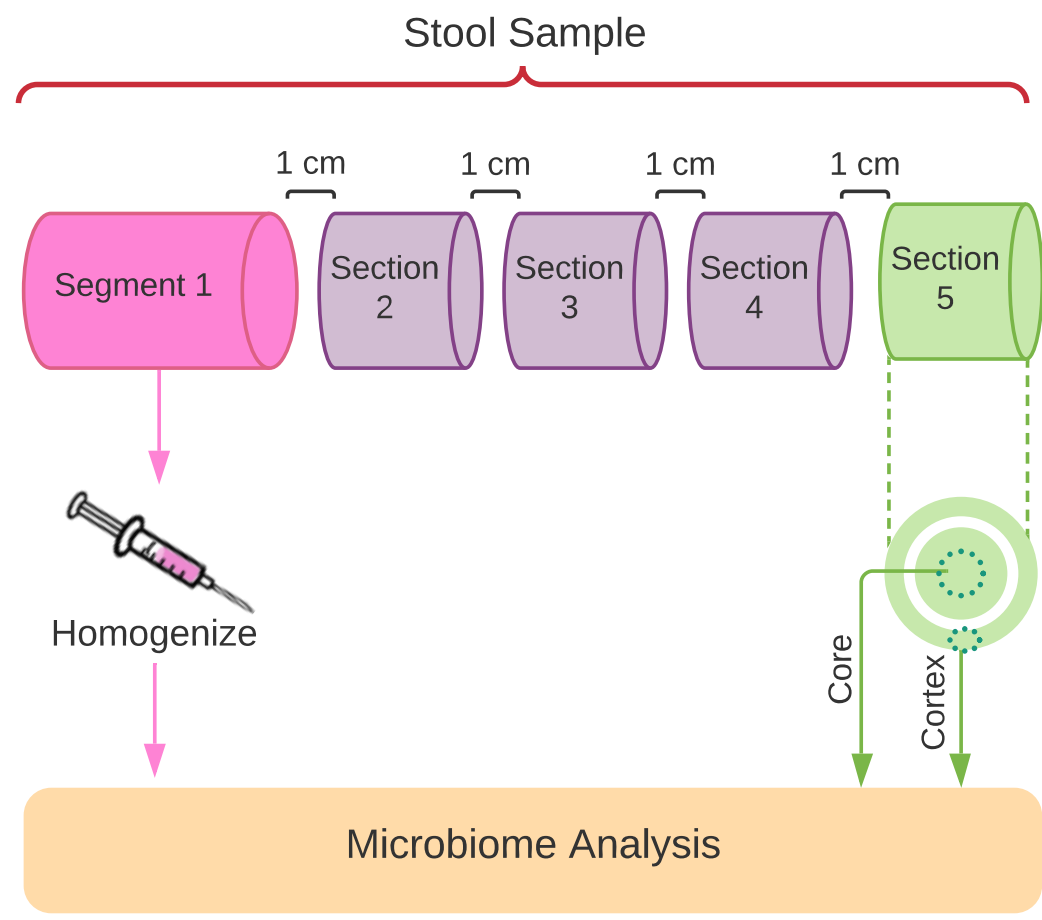

Figure 2. Schematic representation of the stool dissection study to evaluate the level of microbiome homogeneity of each section. Each section was processed independently and analyzed for microbiome composition.

Each section of the stool had dissimilar microbial composition (Figure 3). For example, section 1 was significantly dissimilar $(p<0.01)$ from both the core and cortex of section 6 . There were differences in microbiome composition of each section. Subsamples Replicate aliquots from Section 1, 3, and cortex were all dissimilar from each other while the Segment 1 replicate aliquots were similar. 


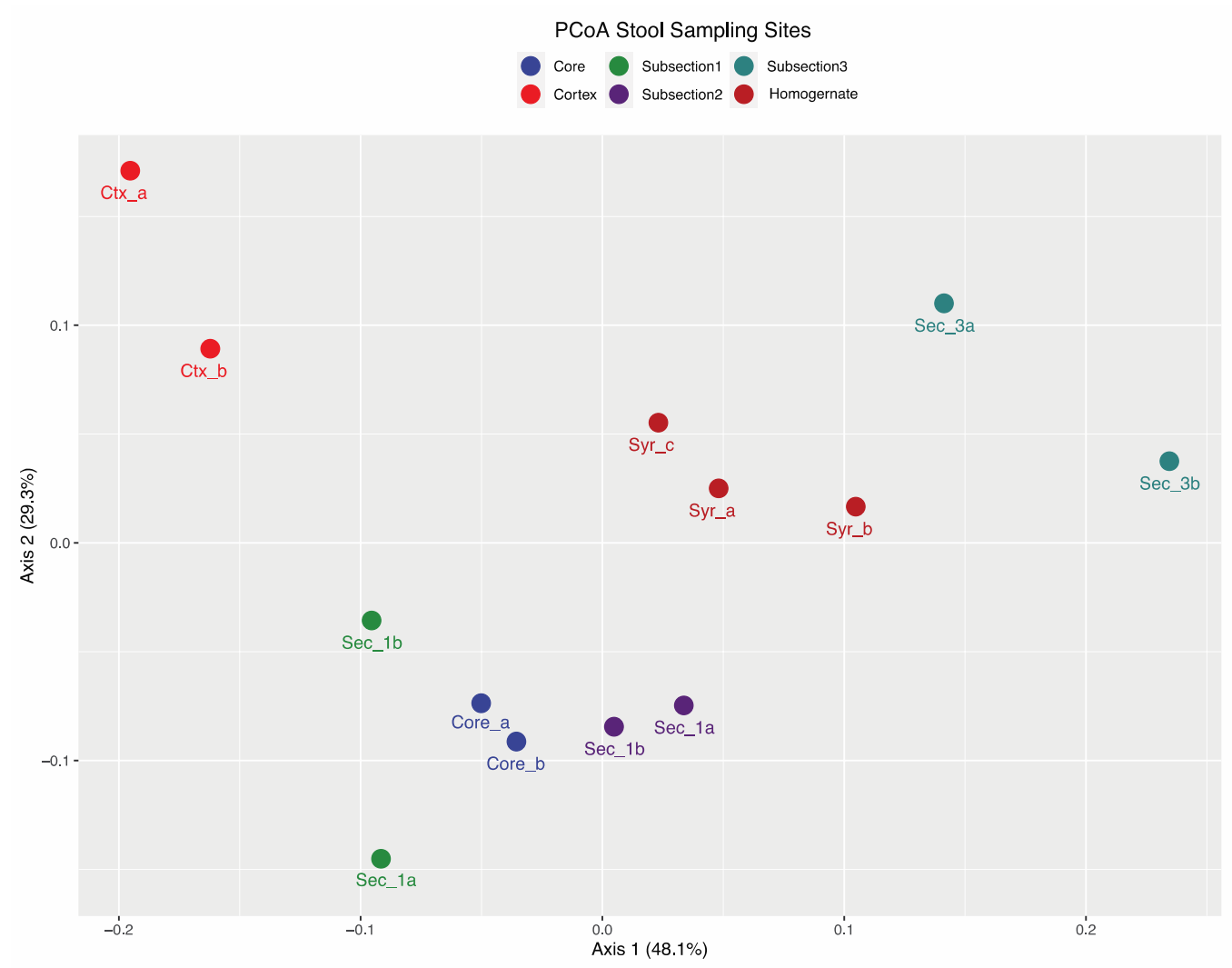

Figure 3. Principal Coordinates Analysis plot based on Bray-Curtis dissimilarity matrix for all stool sub-samples. The PCoA plot shows distinct clustering of the stool samples based on the region or segment of the stool from which they originated. The legend Indicates the 6 different sampling sites, each one $\mathrm{cm}$ apart. The plot illustrates the coordinates for each individual sample.

To illustrate the magnitude of the heterogeneity within a single sample, the relative abundance of key taxa, representing low, medium and high relative abundances across the microbiomes analyzed, was compared in each section (Figure 4). Akkermansia was 3 times more abundant in the cortex compared to the other sections. Alistipes, Bacteriodes and Barnesiella had similar relative abundance profiles to each other in each section. Collinsella and Coprococcus has similar relative abundance profiles in each section. The relative abundance of Bifidobacterium was variable throughout the sections.

The average ratio of Firmicutes:Bacteroidetes was similar for Sections 1, 2, 3 syringe and core ranging from 1.45 to 2.5. However, the Firmicutes:Bacteroidetes ratio was significantly higher at 4.78 for the Cortex making this subsample significantly different from the other sections $(p=0.003)$ and low relative abundance represented in each section of the stool. 

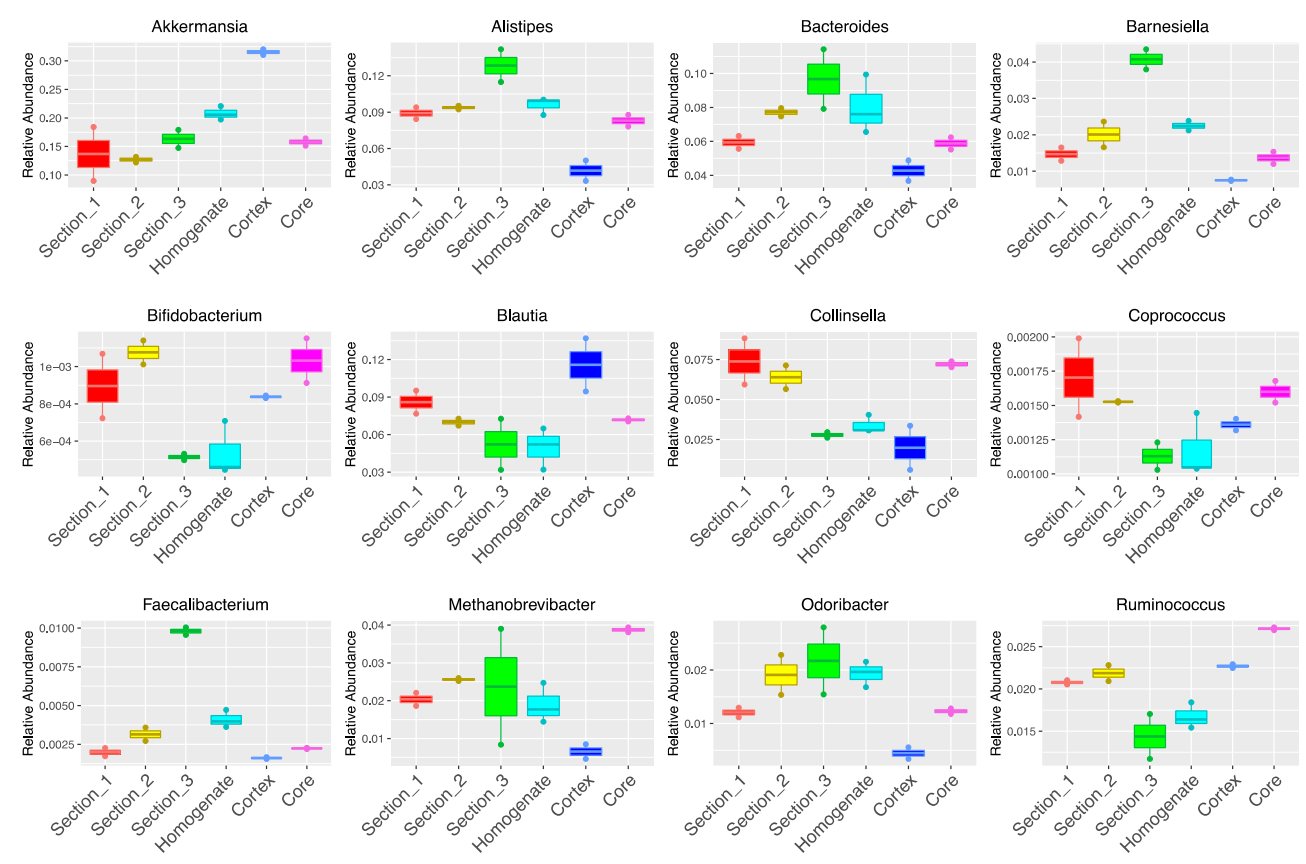

Figure 4. Differential abundance of taxa in dissected stool. The figure summarizes the distribution of 12 different genera present in high, medium

To determine how collection and processing effected microbiome composition, the second bowel movement was collected in the laboratory and processed as shown in Figure 5. The relative abundance (RA) for all phyla in 5 aliquots from each aliquot was compared. There were significant changes in the phyla RA for the fresh subsample while the RA of these five phyla remained stable in the $4 \mathrm{C}$ and frozen subsamples (Figure 5). At T10, Firmicutes and Actinobacteria were highly abundant. Within 2 minutes, the RA of Actinobacteria and Firmicutes began to decrease while the RA of Bacteroidetes and Proteobacteria increased. By 25 minutes, the RA of the Bacteroidetes increased from 0.014 at $\mathrm{T} 10$ to 0.37 at T25, representing a $95.85 \%$ increase. Actinobacteria decreased from 0.21 to 0.04 , an $80 \%$ decrease in 15 minutes (from T10 to T25). Firmicutes decreased from 0.75 to 0.53 or $28.95 \%$. The Proteobacteria increased RA from 0.0112 at T10 to 0.0368 at T25, a $69.6 \%$ increase. There was a significant loss in the Proteobacteria and an increased representation of Actinobacteria in all aliquots of the frozen subsample compared to the fresh and $4 \mathrm{C}$ subsamples $(\mathrm{p}<0.01)$. Proteobacteria RA differed significantly $(\mathrm{p}<0.05)$ between fresh and frozen subsamples. Proteobacteria RA in the frozen subsample averaged $0.0046 \pm 0.0012$, ranging from 0.0035 at $\mathrm{T} 10$ to 0.0045 at $\mathrm{T} 25$. This represents greater than 1 log reduction in RA when compared with the fresh subsample. There were slight variations in RA of the five major phyla in the $4 \mathrm{C}$ aliquots, but these differences were not significant. 


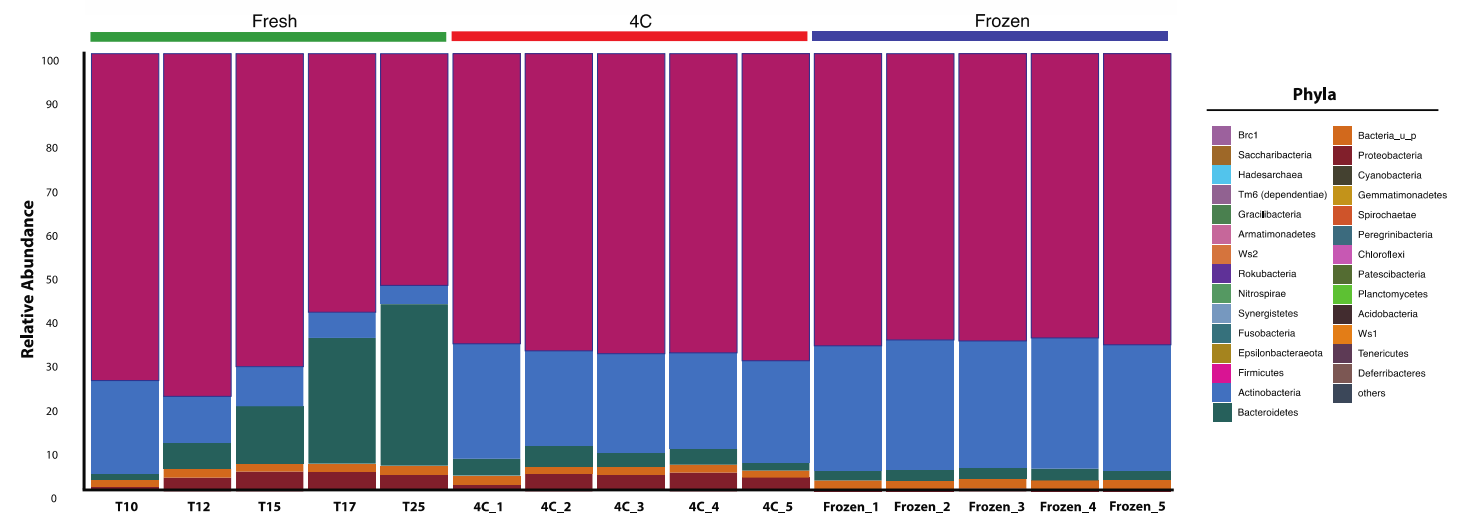

Figure 5. Taxonomic bar graph at the Phylum level for aliquots analyzed from three different sample processing methods. Relative abundance as a percent of total phyla was plotted as a stacked bar graph to illustrate the variations in relative abundance of individual phyla based on sample processing protocol and sampling time.

To determine the effect of processing time on the Firmicutes:Actinobacteria ratio in the stool sample, 5 aliquots taken 10 minutes (T10), 12 minutes (T12), 15 minutes (T15), 17 minutes (T17) and 25 minutes (T25) were sequenced. At T10, Firmicutes and Actinobacteria were highly abundant (Figure 6). Within 2 minutes, the relative abundance (RA) of Actinobacteria and Firmicutes began to decrease while the RA of Bacteroidetes and Proteobacteria increased. At 25 minutes, the RA of the Bacteroidetes increased from 0.014 at T10 to 0.37 at T25, representing a $95.85 \%$ increase. Conversely, the Firmicutes decreased from 0.75 to 0.53 or $28.95 \%$.

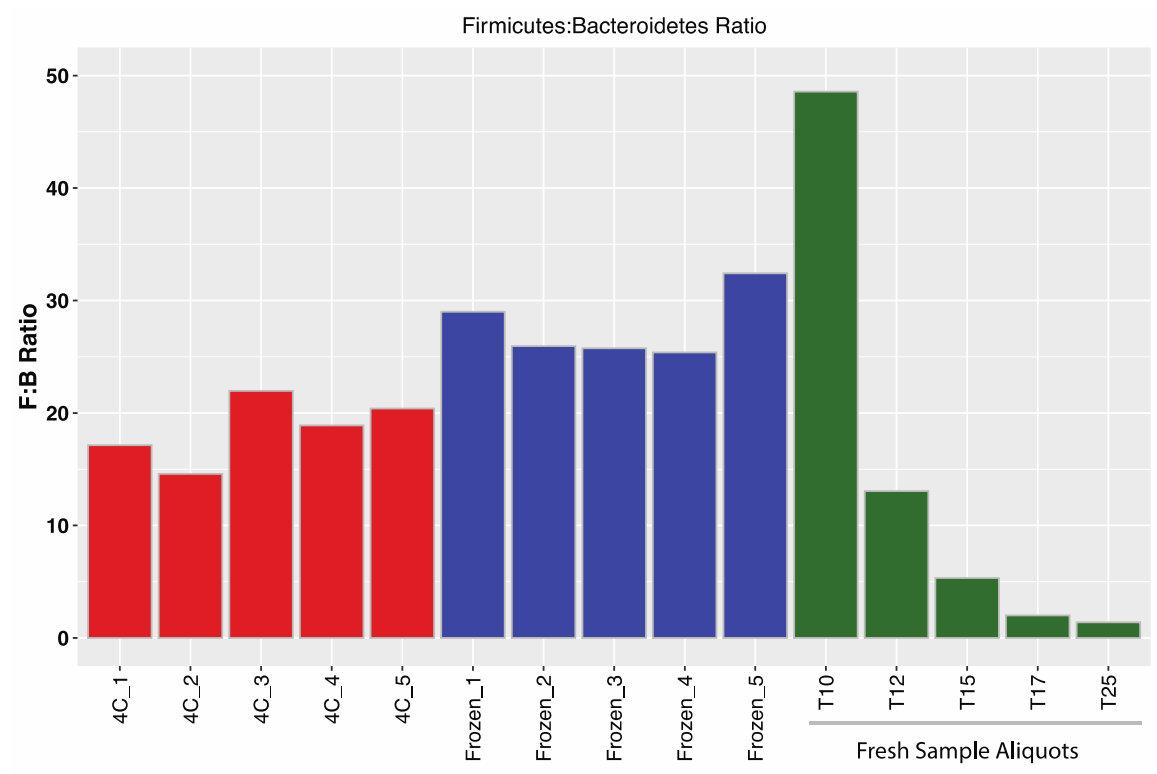

Figure 6. Firmicutes to Bacteroidetes ratio for aliquots analyzed from three different sample processing protocols. The ratio of Firmicutes:Bacteroidetes (F:B) was plotted as a side-by-side bar graph to illustrate the variations in F:B ratio for each sample processing protocol and sampling time. 
Similarly, Actinobacteria decreased from 0.21 to 0.04 , an $80 \%$ decrease in 15 minutes (from T10 to T25). The Proteobacteria showed a similar upward trend in RA to that of the Bacteroidetes. At T10, the calculated RA was 0.0112 and at T25 was 0.0368 or a $69.6 \%$ increase. The ratio of Firmicutes:Bacteroidetes changed from 48:6 at T10 to 1:43 at T25. These results are summarized in Figure 7.

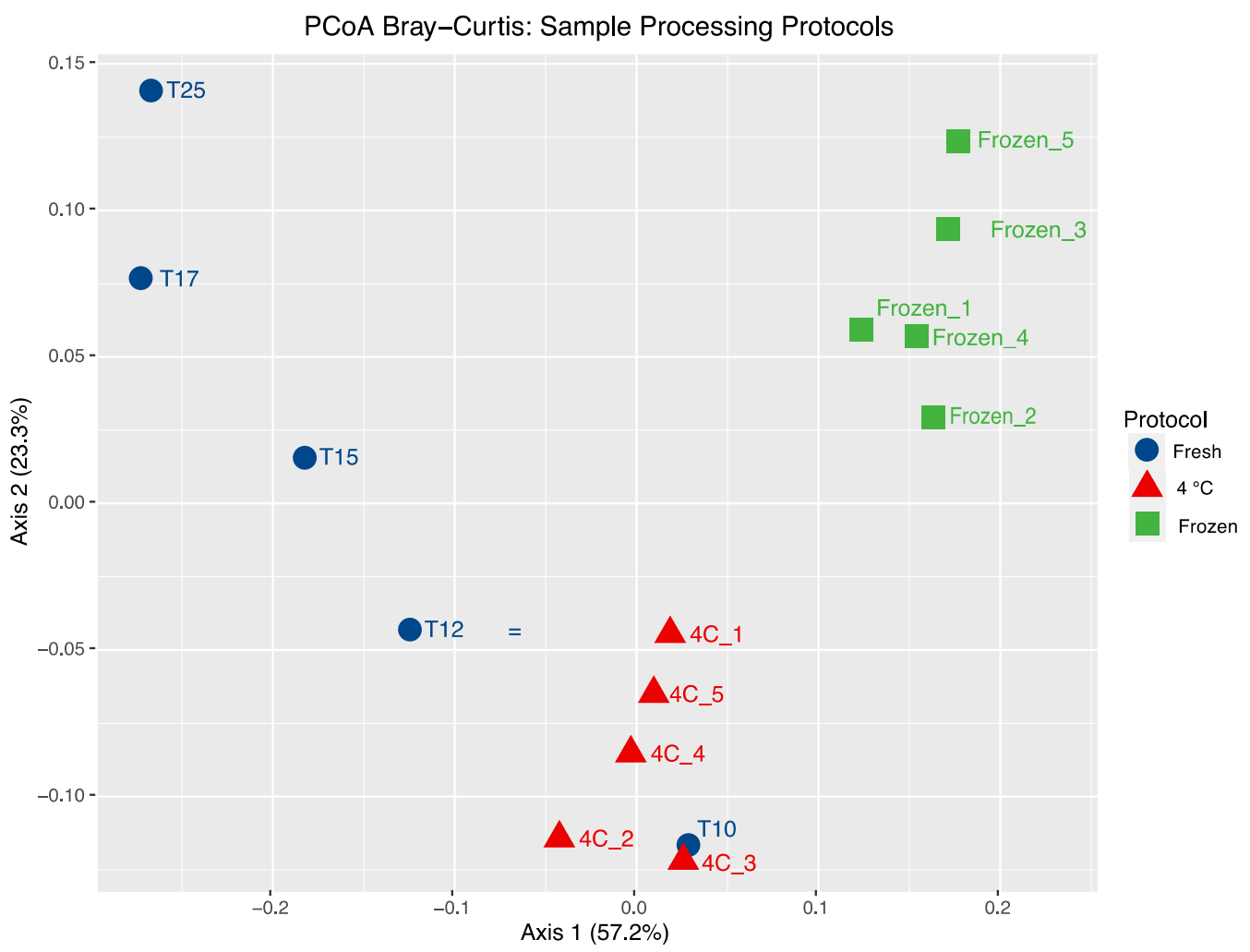

Figure 7. PCoA analysis of for aliquots analyzed from three different sample processing protocols. The PCoA plot shows distinct clustering of the stool samples based on the sample processing protocol used. The legend indicates the 3 different sampling processing protocols used in this study. The plot illustrates the coordinates for each individual sample.

Multidimensional scaling analysis (MDA) [23] using Bray-Curtis distance matrix [24] from QIIME2 workflow [18] was conducted on all replicate samples of the Fresh, 4C and Frozen workflows (Figure 7). The results indicate that both the $4 \mathrm{C}$ and Frozen aliquots group together as cohorts, reflecting the microbiome homogeneity of the aliquots within the cohort. Fresh sample T10, the closest sample to a fresh stool clustered with the 4C cohort. The remaining four aliquots (T12 - T25) progressively separate from the $4 \mathrm{C}$ cohort along Axis 2. 3.2.

The Wilkinson Rank Sum Test for each cohort was calculated and the results indicate that the alpha diversity of the microbiome, as measured using the Shannon and Simpson metrics, were significantly different from each other. The results are summarized in Table 1 
Table 1. Wilkinson Rank Sum Test of alpha diversity and PERMANOVA analysis of beta diversity for the various sample processing protocols

\begin{tabular}{|l|r|r|r|r|}
\hline \multicolumn{2}{|c|}{ Wilcoxon Rank Sum Test } & \multicolumn{2}{c|}{ Simpson } \\
\hline Cohorts & Statistic & \multicolumn{2}{|c|}{ Shannon } & Stiversity) \\
\hline Fresh $\leftrightarrow$-vC & -1.9845 & 0.0472 & -2.6112 & P-value* $^{*}$ \\
\hline Fresh $\leftrightarrow$ Frozen & 1.3578 & 0.1745 & 2.6112 & 0.009 \\
\hline $\mathbf{4 C} \leftrightarrow$ Frozen & 2.6112 & 0.009 & 2.6112 & 0.009 \\
\hline
\end{tabular}

PERMANOVA Analysis (beta diversity)

\begin{tabular}{|c|c|c|}
\hline Cohorts & Statistic & P-value* \\
\hline Fresh $\leftrightarrow 4 \mathrm{C}$ & 1.97 & 0.043 \\
\hline Fresh $\leftrightarrow$ Frozen & 4.433 & 0.006 \\
\hline 4C $\leftrightarrow$ Frozen & 2.567 & 0.005 \\
\hline
\end{tabular}

\section{Discussion}

It is apparent from the results illustrated in Figures 2 and 3 that the human stool, at least in this study, is heterogeneous throughout its length. Such heterogeneity has also been reported in similar, independent studies [29-31]. As such, the microbiome composition can vary depending on the sampling site (e.g., outside surface or inner portions of the stool) as well the location of sampling along the length of the stool. This heterogeneity can be caused by diet, stress, and many other environmental factors [32]. Fecal output in healthy individuals average 1.20 defecations per $24 \mathrm{hr}$ period, with variations from less than one bowel movement per day to more than 2 days per bowel movement, depending upon the transit time of the forming stool [32]. This can significantly affect the composition of the microbiome during formation.

The sampling location of the stool can also have an impact on detection and identification of biomarkers. One salient example is that of bacteria such as Akkermansia muciniphila, a bacterium that predominantly thrive on the mucin layers of the intestinal epithelium, where continuous mucin production by the goblet cells and mucus desquamation occur and promote the growth of this bacterium [33]. This bacterium has been recognized as a biomarker for inflammation [34] and spegut health [35] and suggested for use as a probiotic strain to promote gut health and immunity [36,37] . In our study, it is apparent that the RA of Akkermansia in the fecal cortex $($ mean $=0.54)$ is 3.75 times greater than in samples from the stool's core (mean $=0.16$ ).

Similarly, in our studies, the ratio of Firmicutes to Bacteroidetes (F:B) varied greatly from where the sample was taken and ranged from 5.78 in the stool's cortical sample and 1.12 in Subsample 3 (Figure 3). These data are important to note as the F:B ratio has been used repeatedly as an indicator of gut health and dysbiosis [38, 39].

These results indicate that the null hypothesis, that the stool is homogeneous, is false and as such, fully representative samples must be taken. Fecal samples collected by swabs or wipes would bias the relative abundance of bacteria, depending upon where the swab or wipe sample is taken. To ensure that a representative sample is taken, collection of whole stools with subsequent homogenization can "normalize" the microbiome composition and yield a representative sample from which the microbiome can be evaluated.

The results in Figure 5 indicate cooling the collected whole stool is essential to the stabilization and preservation of the microbiome composition. In this study, a single stool was collected at the laboratory, homogenized at room temperature and immediately split into three groups: Fresh, 4C and Frozen. The fresh group was maintained at room temperature and random aliquots taken for analysis over a period of 25 minutes. The $4 \mathrm{C}$ 
sample was stabilized at $4{ }^{\circ} \mathrm{C}$ then random aliquots were taken over a similar time period. Finally, the Frozen samples were stored in dry ice for 24 hours, thawed at $4{ }^{\circ} \mathrm{C}$ for 24 hours and finally 5 samples taken over a 25 -minute period.

The sample exhibiting the most variability was the Fresh sample. It can be inferred that this sample, which was at "body" temperature when collected, cooled slowly over the 25-minute sampling period at room temperature. During this time, significant changes in key biomarkers such as F:B ratio (Figure 6) along with changes in microbiome composition can be observed (Figure 7). The microbiome composition of the $4{ }^{\circ} \mathrm{C}$ - and dry icestabilized samples exhibited no such dramatic changes in composition over a similar, 25minute sampling period.

Oxygen exposure during sample preparation adversely impacts fecal bacterial communities, primarily the strict anaerobes [40]. Approximately $50 \%$ of bacterial content of stool processed immediately under strict anaerobic conditions is non-viable [41]. Homogenization in ambient air or freeze-thaw reduce viability to $19 \%$ and $23 \%$ respectively. Processing of samples in ambient air can result in several-fold reduction in the abundance of important commensal taxa, including the highly butyrogenic species Faecalibacterium prausnitzii, Subdoligranulum variable, and Eubacterium hallii. The adverse impact of atmospheric oxygen exposure can reduce those species of bacteria nvolved short chain fatty acids (SCFA) biosynthesis. Conversely, while reducing alpha diversity, freeze-thaw does not significantly alter viable microbiota composition [40, 41]. These effects are more notable with stools processed at room temperature than at colder temperatures.

Even brief periods of storage of fecal samples at room temperature can impact the microbiome composition of samples [42]. Gorzelak et al. [42] found significant differences in the major phyla of the gut, Bacteroidetes and Firmicutes, after 30 minutes compared to 15 minutes of storage at room temperature. Our own studies have shown that even 15 minutes at room temperature prior to refrigeration can affect the Firmicutes:Bacteroidetes (F:B) ratio (Figure 6). These changes can occur as a result of differential microbial growth, degradation of genomic DNA present in the stools and/or death of strict anaerobes.

It is essential that the stool be stabilized and the cooling period from fecal deposition to storage be minimized. This effect can be observed as changes in F:B ratios (Figure 7) in samples maintained at room temperature (T10 - T25) and those cooled (4C) or placed in dry ice (Frozen). It is apparent that the F:M ratio changes markedly from T10 - T25 in the fresh samples. It is also notable that when freshly deposited fecal samples are stabilized by placing them immediately on freezer bricks or on dry ice, the F:B ratio remains stable throughout the fecal sample processing steps.

While it can be argued that microbiome analysis involves measuring microbiome DNA composition rather than community viability, DNA resulting from dead or "damaged" anaerobes can be degraded by aerotolerant and facultative anaerobes in the stool, thus, effectively reducing the relative abundance of the anaerobes in subsequent microbiome composition analysis.

It is apparent from the results, that the microbiome of freshly collected stool samples changes in composition rapidly if not stabilized by cooling or freezing as soon as practically possible. It is also apparent, that the method of microbiome stabilization (i.e., $4{ }^{\circ} \mathrm{C}$ or dry ice) has an impact on microbiome composition. Wilkinson Rank Sum tests of alpha diversity metrics (Simpson and Shannon) show significant differences $(p<0.05)$ between the two methods of stabilization (Table 1). When comparing the first two time points (T10 and T12) only of the Fresh sample collection, there is no significant difference in the Wilkinson Rank Sum test between the Fresh (T10 and T12) and the $4{ }^{\circ} \mathrm{C}$ stool stabilization process $(\mathrm{p}=1.21)$. Conversely, when comparing the two methods of stool stabilization (4 ${ }^{\circ} \mathrm{C}$ and dry ice), there is a significant difference between these two processes $(p=0.0090)$. Similarly, PERMANOVA analyses of beta diversity of these two methods also show a significant difference $(\mathrm{p}=0.006)$.

While the stabilization of the stool by rapid cooling is essential for microbiome stabilization and preservation, freezing in dry ice, followed by a period of thawing will cause changes in microbiome composition [43-46], including the four major phyla represented 
in the human gut microbiome (Actinobacteria, Bacteroidetes, Firmicutes and Proteobacteria). While "immediate" freezing has been long considered the best practice for sample preservation for microbiome studies, in our hands, freezing samples in dry ice result in a higher, significant increase $(\mathrm{p}<0.05)$ in Actinobacteria as compared to the $4{ }^{\circ} \mathrm{C}$ sample as well as a higher $\mathrm{F}: \mathrm{B}$ ratio..

\section{Conclusions}

1. The human stool is not homogeneous in microbiome composition and as such, whole stools must be collected to capture the actual microbial composition of the human stool to ensure the integrity of the research, diagnostics and biomarker identification.

2. Collection of the entire bowel movement can be done from the comfort and privacy of the home.

3. Stabilization of the bowel movement at $4{ }^{\circ} \mathrm{C}$ ensures the sample is as representative to the freshly evacuated bowel movement at possible.

4. Fecal subsamples (e.g., swabs) are not representative of the entire gut microbiome and samples that are not collected properly further bias the microbial profile. This is critical since research and biomarker discovery of the gut microbiome requires the availability of provenanced fecal sample that is representative of the gut microbiome and can be used in a variety of platforms. Convenient, at home collection of the entire bowel movement provides sufficient sample to homogenize and aliquot into multiple neat aliquots for testing in genomic, transcriptomic, metabolomic, lipidomic, culturomics, exposomics and phenomics. Although microbial sequencing surveys will continue to advance the field, microbiome research is beginning to focus on the function and mechanistic aspects of microbial communities. Therefore, collection of the entire bowel movement allows study of the microbiome on multiple platforms and concatenation of the all the microbiome data.

Supplementary Materials: The following are available online at www.mdpi.com/xxx/s1, Table S1: "Table_S1_Metadata Table of Dada2 results and Figure S1 "Figure_S1-QCplots.pdf."

Author Contributions: ZZ, DL, MC and RC designed the research study, analyzed the data and wrote the paper. SDV assisted in interpreting the results and writing the paper. All authors read and approved the final

Funding: Please add: Research reported in this publication was supported by the National Institute of General Medical Sciences (NIGMS) of the National Institutes of Health under award number: R44 GM134710-01..

Institutional Review Board Statement: The samples were collected, and the study was conducted according to the guidelines of the Declaration of Helsinki, and approved by the Institutional Review Board wgc IRB, Study Number 1164096 ("The BioCollective: A Crowd-Sourced Marketplace for Microbiome Research") dated 03/23/20."

Informed Consent Statement: The study was approved by Western Institutional Review Board study (20160838) and the study was carried out according to United States federal regulations for the protection of human subjects as codified in 45 CFR 46. Procedures were carried out with the adequate understanding and written consent of the subjects.

Data Availability Statement: The datasets used and/or analyzed are available from the corresponding author upon request.

Acknowledgments: In this section, you can acknowledge any support given which is not covered by the author contribution or funding sections. This may include administrative and technical support, or donations in kind (e.g., materials used for experiments). Our manuscript does not contain any identifiable individual's data in any form

Conflicts of Interest: The authors are employees of The BioCollective, LLC. The company sells The BioCollector, which is designed as a whole stool collection kit. 


\section{References}

1. McDonald, D., et al., American Gut: an open platform for citizen science microbiome research. Msystems, 2018. 3(3): p. e00031-18.

2. Human, T., Project M. A framework for human microbiome research. Nature, 2012. 486(7402): $\mathrm{p}$. 215-21.

3. Sinha, R., et al., Collecting fecal samples for microbiome analyses in epidemiology studies. Cancer Epidemiology and Prevention Biomarkers, 2016. 25(2): p. 407-416.

4. Reyman, M., et al., Rectal swabs are a reliable proxy for faecal samples in infant gut microbiota research based on 16S-rRNA sequencing. Scientific reports, 2019. 9(1): p. 1-8.

5. Biehl, L.M., et al., Usability of rectal swabs for microbiome sampling in a cohort study of hematological and oncological patients. PloS one, 2019. 14(4): p. e0215428.

6. Kumar, R., et al., Getting started with microbiome analysis: sample acquisition to bioinformatics. Current protocols in human genetics, 2014. 82: p. 18.8.1-18.8.29.

7. Dubois, G., et al., The Inuit gut microbiome is dynamic over time and shaped by traditional foods. Microbiome, 2017. 5(1): p. 1-12.

8. Kurian, S.M., et al., Feasibility and Comparison Study of Fecal Sample Collection Methods in Healthy Volunteers and Solid Organ Transplant Recipients Using 16S rRNA and Metagenomics Approaches. Biopreservation and Biobanking, 2020.

9. Wu, W.-K., et al., Optimization of fecal sample processing for microbiome study - The journey from bathroom to bench. Journal of the Formosan Medical Association, 2019. 118(2): p. 545-555.

10. Zackular, J.P., et al., The human gut microbiome as a screening tool for colorectal cancer. Cancer prevention research, 2014. 7(11): p. 1112-1121.

11. Costea, P.I., et al., Towards standards for human fecal sample processing in metagenomic studies. Nat Biotechnol, 2017. 35(11): p. 1069-1076.

12. Vogtmann, E., et al., Comparison of collection methods for fecal samples in microbiome studies. American journal of epidemiology, 2017. 185(2): p. 115-123.

13. Watson, E.-J., et al., Human faecal collection methods demonstrate a bias in microbiome composition by cell wall structure. Scientific reports, 2019. 9(1): p. 1-8.

14. Wu, W.K., et al., Optimization of fecal sample processing for microbiome study - The journey from bathroom to bench. J Formos Med Assoc, 2018.

15. Sutherland, V.L., et al., The Gut Microbiome and Xenobiotics: Identifying Knowledge Gaps. Toxicological Sciences, 2020. 176(1): p. 1-10.

16. Lewis, S.J. and K.W. Heaton, Stool form scale as a useful guide to intestinal transit time. Scandinavian journal of gastroenterology, 1997. 32(9): p. 920-924.

17. Callahan, B.J., et al., DADA2: high-resolution sample inference from Illumina amplicon data. Nature methods, 2016. 13(7): p. 581-583.

18. Bolyen, E., et al., Reproducible, interactive, scalable and extensible microbiome data science using QIIME 2. Nature biotechnology, 2019. 37(8): p. 852-857.

19. Quast, C., et al., The SILVA ribosomal RNA gene database project: improved data processing and web-based tools. Nucleic acids research, 2012. 41(D1): p. D590-D596.

20. Shannon, C.E., A mathematical theory of communication. ACM SIGMOBILE mobile computing and communications review, 2001. 5(1): p. 3-55.

21. Chao, A., Nonparametric estimation of the number of classes in a population. Scandinavian Journal of statistics, 1984: p. 265-270.

22. Pielou, E.C., The measurement of diversity in different types of biological collections. Journal of theoretical biology, 1966. 13: p. 131-144.

23. Cox, M.A.A. and T.F. Cox, Multidimensional scaling, in Handbook of data visualization. 2008, Springer. p. 315-347.

24. Bray, J.R. and J.T. Curtis, An ordination of upland forest communities of southern Wisconsin. Ecological Monographs (27). 1957, PRIMER-E Plymouth. p. 325-349.

25. Jaccard, P., Étude comparative de la distribution florale dans une portion des Alpes et des Jura. Bull Soc Vaudoise Sci Nat, 1901. 37: p. 547-579.

26. Mann, H.B. and D.R. Whitney, On a test of whether one of two random variables is stochastically larger than the other. The annals of mathematical statistics, 1947: p. 50-60.

27. Anderson, M.J., A new method for non-parametric multivariate analysis of variance. Austral ecology, 2001. 26(1): p. 32-46.

28. Daniel, W.W., Kruskal-Wallis one-way analysis of variance by ranks. Applied nonparametric statistics, 1990: p. 226-234.

29. Wu, G.D., et al., Sampling and pyrosequencing methods for characterizing bacterial communities in the human gut using 16S sequence tags. BMC Microbiol, 2010. 10: p. 206. 
30. Huson, D.H., et al., A simple statistical test of taxonomic or functional homogeneity using replicated microbiome sequencing samples. Journal of biotechnology, 2017. 250: p. 45-50.

31. Gratton, J., et al., Optimized sample handling strategy for metabolic profiling of human feces. Analytical chemistry, 2016. 88(9): p. 4661-4668.

32. Rose, C., et al., The Characterization of Feces and Urine: A Review of the Literature to Inform Advanced Treatment Technology. Crit Rev Environ Sci Technol, 2015. 45(17): p. 1827-1879.

33. Van Herreweghen, F., et al., Mucin degradation niche as a driver of microbiome composition and Akkermansia muciniphila abundance in a dynamic gut model is donor independent. FEMS microbiology ecology, 2018. 94(12): p. fiy186.

34. Ouyang, J., et al., The Bacterium Akkermansia muciniphila: A Sentinel for Gut Permeability and Its Relevance to HIV-Related Inflammation. Frontiers in immunology, 2020. 11: p. 645-645.

35. Jayachandran, M., S.S.M. Chung, and B. Xu, A critical review of the relationship between dietary components, the gut microbe Akkermansia muciniphila, and human health. Critical reviews in food science and nutrition, 2020. 60(13): p. 2265-2276.

36. Zhang, T., et al., Akkermansia muciniphila is a promising probiotic. Microbial biotechnology, 2019. 12(6): p. 1109-1125.

37. Ansaldo, E., et al., Akkermansia muciniphila induces intestinal adaptive immune responses during homeostasis. Science, 2019. 364(6446): p. 1179-1184.

38. Magne, F., et al., The Firmicutes/Bacteroidetes Ratio: A Relevant Marker of Gut Dysbiosis in Obese Patients? Nutrients, 2020. 12(5): p. 1474.

39. Jasirwan, C.O.M., et al., Correlation of Gut Firmicutes/Bacteroidetes Ratio with Fibrosis and Steatosis in Patients with Non-alcoholic Fatty Liver Disease. 2020.

40. Chu, N.D., et al., Profiling living bacteria informs preparation of fecal microbiota transplantations. PloS one, 2017. 12(1): p. e0170922.

41. Papanicolas, L.E., et al., Bacterial viability in faecal transplants: which bacteria survive? EBioMedicine, 2019. 41: p. 509-516.

42. Gorzelak, M.A., et al., Methods for Improving Human Gut Microbiome Data by Reducing Variability through Sample Processing and Storage of Stool. PLoS One, 2015. 10(8): p. e0134802.

43. Molbak, L., et al., Freezing at- $80^{\wedge}$ oC Distorts the DNA Composition of Bacterial Communities in Intestinal Samples. Current Issues in Intestinal Microbiology, 2006. 7(1): p. 29.

44. Walker, V.K., G.R. Palmer, and G. Voordouw, Freeze-thaw tolerance and clues to the winter survival of a soil community. Applied and Environmental Microbiology, 2006. 72(3): p. 1784-1792.

45. Choo, J.M., L.E. Leong, and G.B. Rogers, Sample storage conditions significantly influence faecal microbiome profiles. Sci Rep, 2015. 5: p. 16350.

46. Ezzy, A.C., et al., Storage and handling of human faecal samples affect the gut microbiome composition: A feasibility study. Journal of microbiological methods, 2019. 164: p. 105668. 